\title{
Impact of Early Surgical Evacuation of Sylvian Hematoma on Clinical Course and Outcome After Subarachnoid Hemorrhage
}

\author{
Tatsushi MUTOH, Tatsuya ISHIKAWA, Junta MOROI, \\ Akifumi SUZUKI, and Nobuyuki YASUI
}

Department of Surgical Neurology, Research Institute for Brain and Blood Vessels-Akita, Akita

\begin{abstract}
The present study aimed to evaluate the impact of early surgical evacuation of sylvian hematoma caused by ruptured middle cerebral artery (MCA) aneurysm on clinical outcome after subarachnoid hemorrhage. Hospital records and computed tomography scans for 26 patients with MCA aneurysm who underwent surgical clipping between June 2001 and January 2008 were retrospectively reviewed. All patients presented with sylvian hematoma associated with subarachnoid hemorrhage and received surgery at $7.9 \pm 3.6$ (mean \pm standard deviation) hours of ictus. They were divided postoperatively into two groups, achievement $(n=16)$ and non-achievement $(n=10)$ of extensive hematoma evacuation, and their clinical course and functional outcomes were compared. The frequencies of delayed ischemic neurological deficit and vasospasm-related cerebral infarction were significantly less $(p<0.05)$ in the achievement group. Better functional outcomes were obtained in patients with successful evacuation (p $<\mathbf{0 . 0 5}$ ), as assessed by improvement of hemiparesis on manual muscle testing scale at postoperative 1month follow up and by the modified Rankin scale at postoperative 3 and 6 months. Clinical outcomes were also better in the achievement group. These results suggest that better clinical course and outcome can be expected in patients who undergo early successful hematoma evacuation with surgical clipping of a ruptured MCA aneurysm.
\end{abstract}

Key words: clinical outcome, early surgical evacuation, middle cerebral artery, ruptured aneurysm, sylvian hematoma

\section{Introduction}

Dense focal hematoma is frequently observed in the sylvian fissure and/or the temporal lobe after rupture of middle cerebral artery (MCA) aneurysm. Sylvian hematoma is a negative prognostic factor associated with mortality rates of $21 \%$ to $85 \%$, even after successful aneurysm clipping and simultaneous hematoma evacuation. ${ }^{9,10,16,17,21,25)}$ Patients with sylvian hematoma have higher rates of perioperative complications and poorer outcomes than those with temporal lobe intracerebral hematoma (ICH) from ruptured MCA aneurysms. ${ }^{17,21)}$ Emergency surgery within 6 to 12 hours of bleeding onset is particularly indicated in cases of sylvian hematoma to prevent irreversible brain damage and serious cerebral edema associated with progression of hematoma. ${ }^{1,21)}$ However, clinical results to date have been unsatisfactory, possibly due to technical difficulties in early surgical removal arising from increased intracranial pressure (ICP) and subarachnoid adhesions if the clots are very firm and massively distributed over the distal sylvian fissure or within the pia mater. Such residual hematoma may result in rebleeding, subsequent brain edema, and symptomatic cerebral vasospasm. ${ }^{17,23,27)}$ We recently proposed a strategy to improve the treatment outcome of subarachnoid hemorrhage (SAH) patients with sylvian hematoma associated with ruptured MCA aneurysms, comprising early surgical evacuation prior to hematoma expansion; extensive removal of any dense subarachnoid and subpial clots extending to the anterior, posterior, and superior limiting sulcus of the insular cortex; and coagulation of bleeding pial vessels injured by the impact of aneurysm rupture. ${ }^{12,24)}$

The present single-center, retrospective study analyzed our clinical experience with this strategy in combination with aneurysm clipping with regard to the clinical course and outcome after SAH. 


\section{Materials and Methods}

\section{Patient population}

Medical records and computed tomography (CT) scans for all patients with acutely ruptured intracranial aneurysms presenting to the emergency stroke service and admitted to the neurosurgical intensive care unit at our institute between June 2001 and January 2008 were reviewed. Inclusion criteria comprised: the presence of sylvian hematoma verified on admission CT scan, the presence of MCA aneurysm as demonstrated by CT angiography or digital subtraction angiography (DSA), and surgical clipping of the ruptured aneurysm performed within 24 hours of symptom onset (designated study day 0 ). From a total of $280 \mathrm{SAH}$ cases treated during the designated period, we identified 26 patients (15 women and 11 men; age, $64 \pm 8$ yrs) with dense clot

Table 1 Clinical characteristics of 26 patients with sylvian hematoma after SAH

\begin{tabular}{lcc}
\hline & \multicolumn{2}{c}{ Evacuation achieved } \\
\cline { 2 - 3 } & Yes & No \\
\hline No. of patients & 16 & 10 \\
Sex, male/female & $7 / 9$ & $4 / 6$ \\
Age, yrs & $62 \pm 8$ & $64 \pm 9$ \\
WFNS grade & & \\
I & 2 & 1 \\
III & 3 & 6 \\
IV & 9 & 1 \\
V & 2 & 2 \\
Side of MCA aneurysm, right/left & $10 / 6$ & $6 / 4$ \\
Hematoma distribution, $\Delta$ post-pre (cm) & & \\
A & $-0.9 \pm 1.0^{*}$ & $-0.3 \pm 0.5$ \\
P & $-1.8 \pm 1.0^{*}$ & $0.0 \pm 0.5$ \\
S & $0.6 \pm 0.9^{*}$ & $0.4 \pm 0.5$ \\
Hematoma volume, ml & $26 \pm 7$ & $24 \pm 8$ \\
Decompressive surgery, n (\%) & $2(13)^{*}$ & $5(50)$ \\
Time from onset to surgery, hrs & $8.4 \pm 4.1$ & $7.1 \pm 2.4$ \\
Length of hospital stay, days & $42 \pm 20$ & $58 \pm 34$ \\
Angiographic vasospasm, n (\%) & $8(50)$ & $7(70)$ \\
DIND, n (\%) & $4(25)^{*}$ & $6(60)$ \\
Infarction, n (\%) & $2(13)^{*}$ & $4(40)$ \\
VP shunt for NPH, n (\%) & $4(25)$ & $2(20)$ \\
MMT score, $\Delta$ post-pre & $2.1 \pm 1.8^{*}$ & $-1.3 \pm 1.4$ \\
mRS score & & \\
at 3 months & $2.2 \pm 1.4^{*}$ & $4.7 \pm 0.9$ \\
at 6 months & $1.5 \pm 1.3^{*}$ & $4.2 \pm 1.1$ \\
\hline
\end{tabular}

${ }^{*} \mathrm{p}<0.05$ vs. non-evacuation group. A, P, and S: hematoma distribution along the anterior, posterior, and superior limiting sulcus of the insular cortex, respectively; DIND: delayed ischemic neurological deficit; VP: ventriculoperitoneal; $\Delta$ post-pre: postoperative value minus preoperative value; MCA: middle cerebral artery; MMT: manual muscle testing; mRS: modified Rankin scale; NPH: normal pressure hydrocephalus; WFNS: World Federation of Neurosurgical Societies. distribution within the sylvian fissure (Fisher CT scale $^{5)}$ grade 3) associated with a ruptured MCA aneurysm. Patient characteristics are presented in Table 1.

\section{Definition and assessment of sylvian hemato- ma}

Patients were divided into two groups, achievement or non-achievement of hematoma evacuation, based on CT findings of pre- and postoperative hematoma distribution. Extension of sylvian hematoma was divided into anterior, posterior, and superior directions along the limiting sulcus of the insular cortex (Fig. 1A), and was scored based on distribution $(\mathrm{cm})$ in the anterior and posterior directions and hematoma thickness (defined as the maximum diameter at the level of corona radiata) in the superior direction. Hematoma was considered as residual if postoperative CT still showed localized
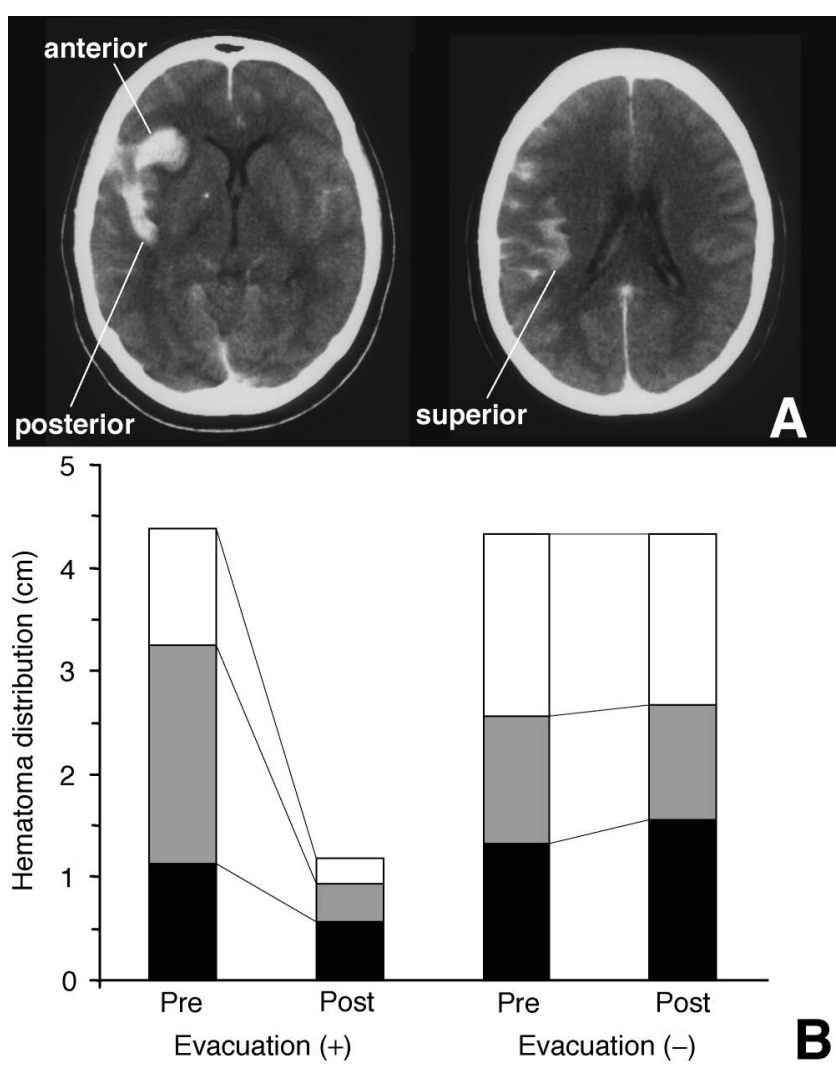

Fig. 1 A: Preoperative computed tomography scans showing extension of sylvian hematoma. B: Graphs demonstrating preoperative and postoperative changes ( $\Delta \mathrm{cm}$, mean values) in hematoma extension in anterior (open columns), posterior (shaded columns), and superior (closed columns) directions along the limiting sulcus of the insular cortex in the groups of achievement $(n=16)$ and non-achievement $(n=10)$ of hematoma evacuation. 
hematoma $>2 \mathrm{~cm}$ in either distribution or thickness. Hematoma volumes were determined preoperatively using semiautomated segmentation and/or freehand tracing tools in the region of interest (ROI) module with software (OsiriX) running on a Macintosh computer (Apple Inc, Cupertino, Calif., U.S.A.). ROI volume was calculated by multiplying the thickness of the acquired section by the area. ${ }^{19)}$ Due to the inconsistencies in the calculation of postoperative hematoma volume (variability in clot densities and associated difficulties in freehand tracing), assessment of hematoma evacuation in this study was quantified using preoperative and postoperative change $(\Delta \mathrm{cm})$ in hematoma extension in each direction along the limiting sulcus of the insular cortex.

\section{Evacuation procedure}

The extent of clot removal was decided by the operating surgeon, based primarily on technical difficulties associated with firmness of hematoma clots, surgical instrumentation, and experience with hematoma evacuation procedures. Intraoperative fibrinolysis for subarachnoid clots, hematoma, and cisterns was performed in all cases by intermittent irrigation with tissue plasminogen activator (Cleactor; Eisai Co. Ltd., Tokyo at a concentration of $100,000 \mathrm{IU}$ in $500 \mathrm{ml}$ of $0.9 \%$ saline solution. A highpressure irrigation system (Suction Plus; Codman, Johnson \& Johnson Co. Ltd., Tokyo) was used to assist with irrigation and suction. Extensive removal of subarachnoid and subpial clots was performed as far as possible, extending to the limiting sulcus of the insular cortex and coagulated injured subpial vessels projecting to the hematoma without damaging the sylvian veins to prevent further rebleeding and associated hematoma expansion.

\section{Clinical management}

Immediately after brief neurological and systemic preoperative examination, patients were deeply sedated to prevent aneurysm rerupture. After verification of the aneurysm on DSA, aneurysm clipping combined with simultaneous hematoma evacuation was performed via pterional craniotomy with a distal transsylvian approach. At the end of the operation, the cisterns were irrigated systematically and a cisternal drain was inserted for postoperative cerebrospinal fluid drainage. This was usually removed within 5 days, but spinal drainage was continued after removal of the cisternal drain when necessary to control ICP. Such drainage was tapered off until removal at postoperative day 14 or otherwise converted to a ventriculoperitoneal (VP) shunt for normal pressure hydrocephalus.

Patients were followed up with daily transcranial
Doppler (TCD) over the temporal bone windows using standard criteria for angiographic vasospasm. ${ }^{20}$ On postoperative days 7 and 14, routine screening with diffusion-weighted magnetic resonance (MR) imaging with MR angiography and stable technetium-99m hexamethylpropyleneamine oxime single photon emission computed tomography was performed for evaluation of post-SAH vasospasm. If mean blood flow velocity was $>120 \mathrm{~cm} / \mathrm{sec}$ or had increased by $50 \mathrm{~cm} / \mathrm{sec}$ on TCD within 24 hours, the schedule for imaging studies was brought forward. Delayed ischemic neurological deficit was defined as worsening of neurological condition that could not be attributed to systemic or postoperative complications. Patients who become symptomatic with delayed ischemic neurological deficit due to vasospasm were placed on a continuous intravenous infusion of dobutamine under the standard hyperdynamic therapy approach to medical management of cerebral vasospasm at our institute. ${ }^{6)}$

\section{Data analysis}

The following information was recorded for each patient: age, sex, World Federation of Neurosurgical Societies (WFNS) grade, ${ }^{4)}$ side of aneurysm, time from symptom onset to surgery, requirement for decompressive surgery and VP shunt surgery, distribution and volume of hematoma, hemiparesis, vasospasm, and outcome. The incidences of TCDdetected angiographic vasospasm and delayed ischemic neurological deficit, occurrence of infarction attributed to vasospasm, changes in hemiparesis on the side affected by the hematoma between admission and at 1 month as assessed by manual muscle testing (MMT) (rating scale: 5, Patient can hold position against maximum resistance and through complete range of motion; 4, Patient can hold position against strong to moderate resistance, with full range of motion; 3, Patient can tolerate no resistance but can perform the movement through the full range of motion; 2, Patient has all or partial range of motion in the position with gravity eliminated; 1 , The muscle/muscles can be palpated while the patient is performing the action in the position with gravity eliminated; and 0 , No contractile activity can be felt in the position with gravity eliminated), ${ }^{11)}$ and functional outcomes at postoperative 3 and 6 months determined according to the modified Rankin scale (mRS; 0, full recovery-6, death; poor outcome defined as death or severe disability [4$6])^{26)}$ were evaluated. For comparisons of hematoma distribution, MMT and mRS, differences $(\Delta$ post-pre) between each variable before (on admission) and after surgery (on initial examination after full recovery from anesthesia) were analyzed. 
Statistical analysis was performed using software (GraphPad PRISM version 5.01; GraphPad Software, San Diego, Calif., U.S.A.). A Gaussian distribution could not be assumed because of the relatively small sample size. Therefore, statistical analysis was performed with the two-tailed Mann-Whitney U test. Categorical comparisons were made using the Fisher's exact test. A $p$ value $<0.05$ was considered statistically significant. All values are expressed as mean \pm standard deviation, unless otherwise stated.

\section{Results}

Extensive evacuation of sylvian hematoma was achieved following surgical clipping of a ruptured
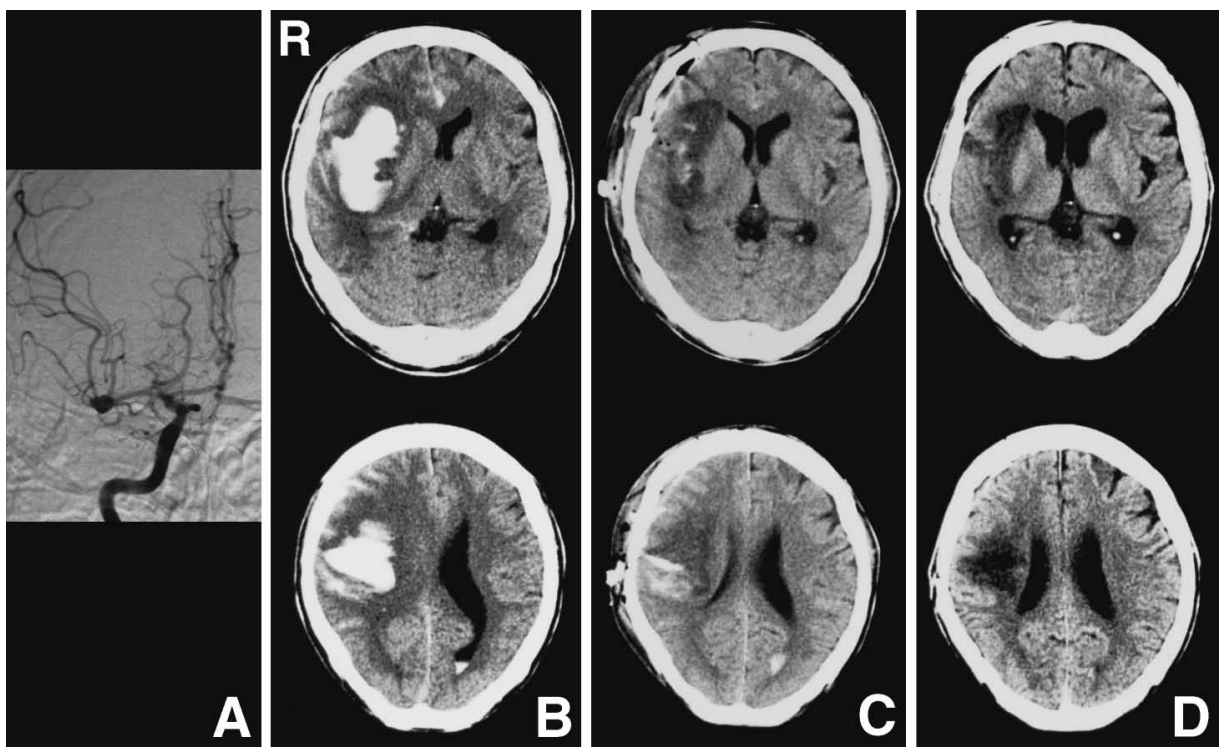

Fig. 2 Achievement of evacuation of sylvian hematoma. A: Preoperative digital subtraction angiogram showing a middle cerebral artery aneurysm on the right. B-D: Preoperative (B), 12-hour (C) and 1-month (D) postoperative computed tomography scans.
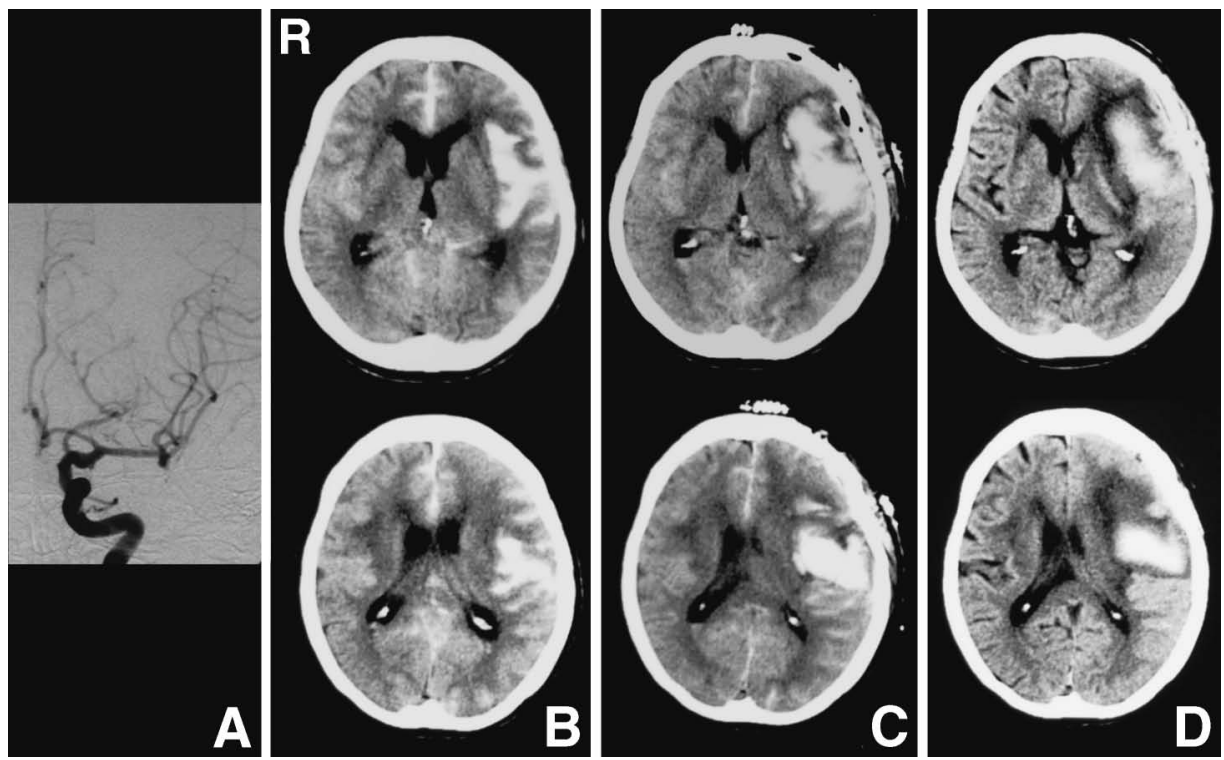

Fig. 3 Postoperative residual sylvian hematoma and its expansion. A: Preoperative digital subtraction angiogram showing a middle cerebral artery aneurysm on the left. B-D: Preoperative (B), 12-hour (C) and 1-month (D) postoperative computed tomography scans. 

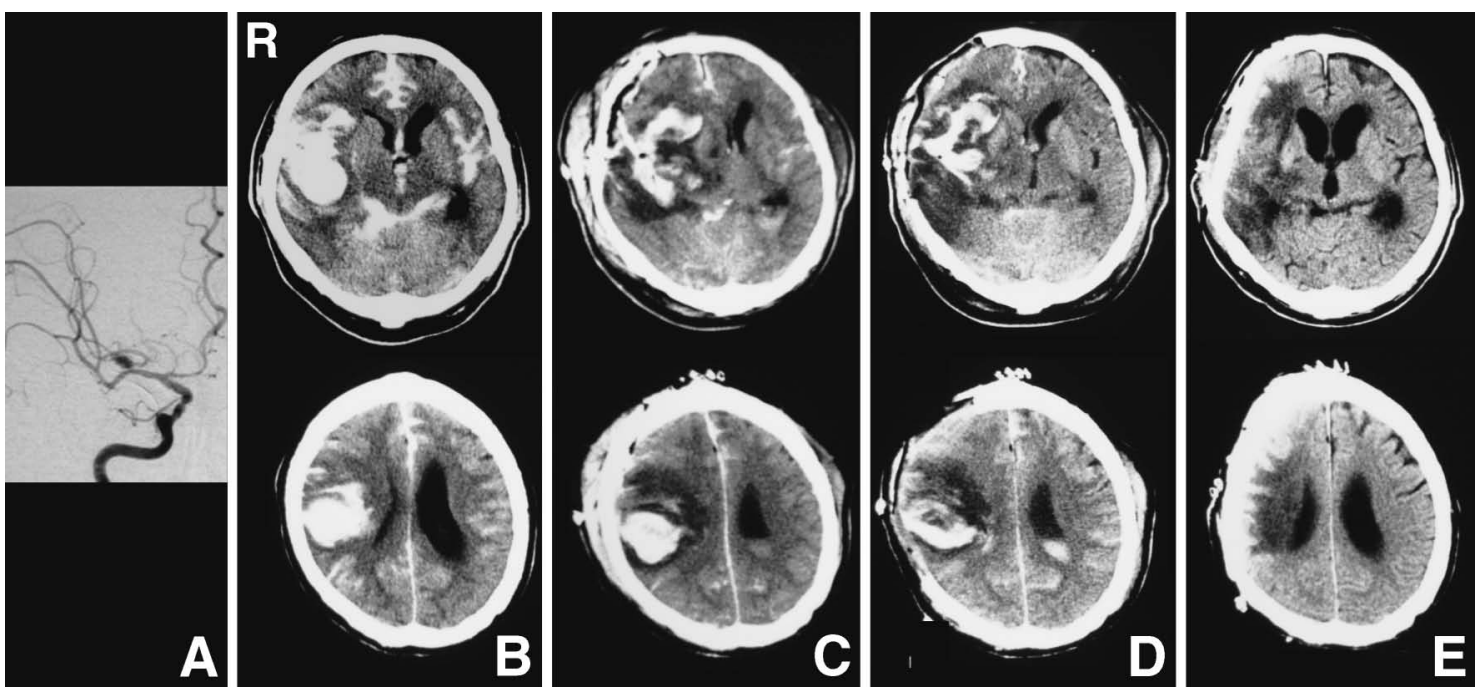

Fig. 4 Postoperative residual hematoma and expansion of perifocal edema with mass effect. A: Preoperative digital subtraction angiogram showing a middle cerebral artery aneurysm on the right. B-E: Preoperative (B), 12-hour (C), 3-day (day after decompressive craniectomy) (D) and 1-month (E) postoperative computed tomography scans demonstrating postoperative residual hematoma and expansion of perifocal edema with mass effect.

MCA aneurysm in 16 patients (Fig. 2). However, residual hematoma $(\geq 2 \mathrm{~cm})$ was evident in 10 patients according to the criteria for postoperative CT findings (Fig. 3). These patients had thick intrasylvian hematoma that could not be removed easily due to firm clot around the subpial vessels, and 2 of the 10 patients also exhibited postoperative hematoma expansion. A total of 3 patients required second surgery for decompressive craniectomy due to postoperative hematoma expansion or severe brain swelling without hematoma expansion (Fig. 4). No patients with successful evacuation suffered such severe postoperative events.

The averaged pre- and postoperative distributions of sylvian hematoma are shown in Fig. 1B and Table 1. There were no significant differences in preoperative distribution of hematoma between the groups with and without successful hematoma evacuation ( $p>0.05$ for all directions). In the successful evacuation group, hematoma was most effectively removed in the posterior limiting sulcus of the insular cortex $(\mathrm{p}<0.05)$ (Table 1$)$.

There were no significant differences between the achievement and non-achievement of hematoma evacuation groups regarding admission data, including age, sex, WFNS grade, side of aneurysms, and time to surgery ( $p>0.05$ for all parameters). Decompression with craniectomy and duraplasty using the pericranium was performed simultaneously in 4 patients $(2$ in the achievement group, 2 in the nonachievement group; $p=0.50$ ) due to brain swelling at surgical closure preventing restoration of the

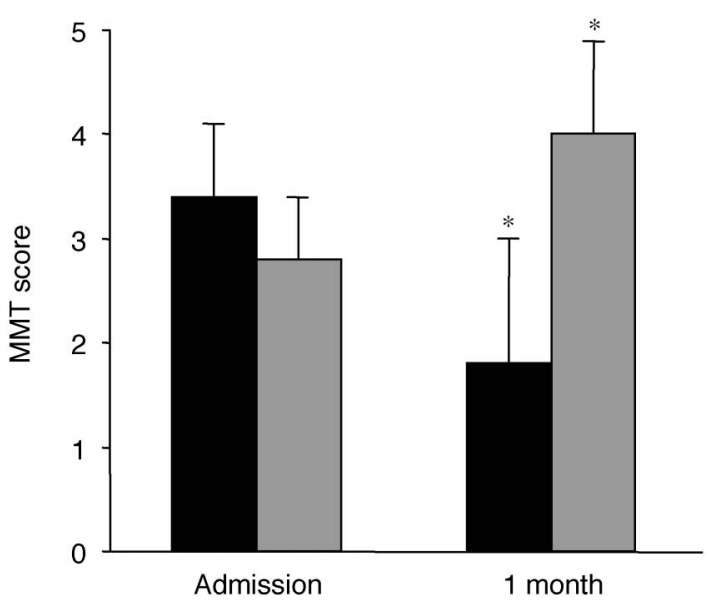

Fig. 5 Changes in motor function on the side affected by aneurysm rupture and associated hematoma according to manual muscle testing (MMT) score from initial presentation to discharge. ${ }^{*} p<0.05$ vs. MMT score on admission. Closed columns: evacuation $(+)$, shaded columns: evacuation $(-)$.

bone flap. Normal pressure hydrocephalus requiring VP shunt surgery developed in the chronic stage in $23 \%(n=6)$ of all patients, with no significant difference in incidence between the groups $(p=0.13)$ (Table 1).

The incidences of angiographic vasospasm, delayed ischemic neurological deficits $(p=0.03)$, and associated infarction $(p=0.03)$ tended to be lower in patients with successful hematoma evacua- 

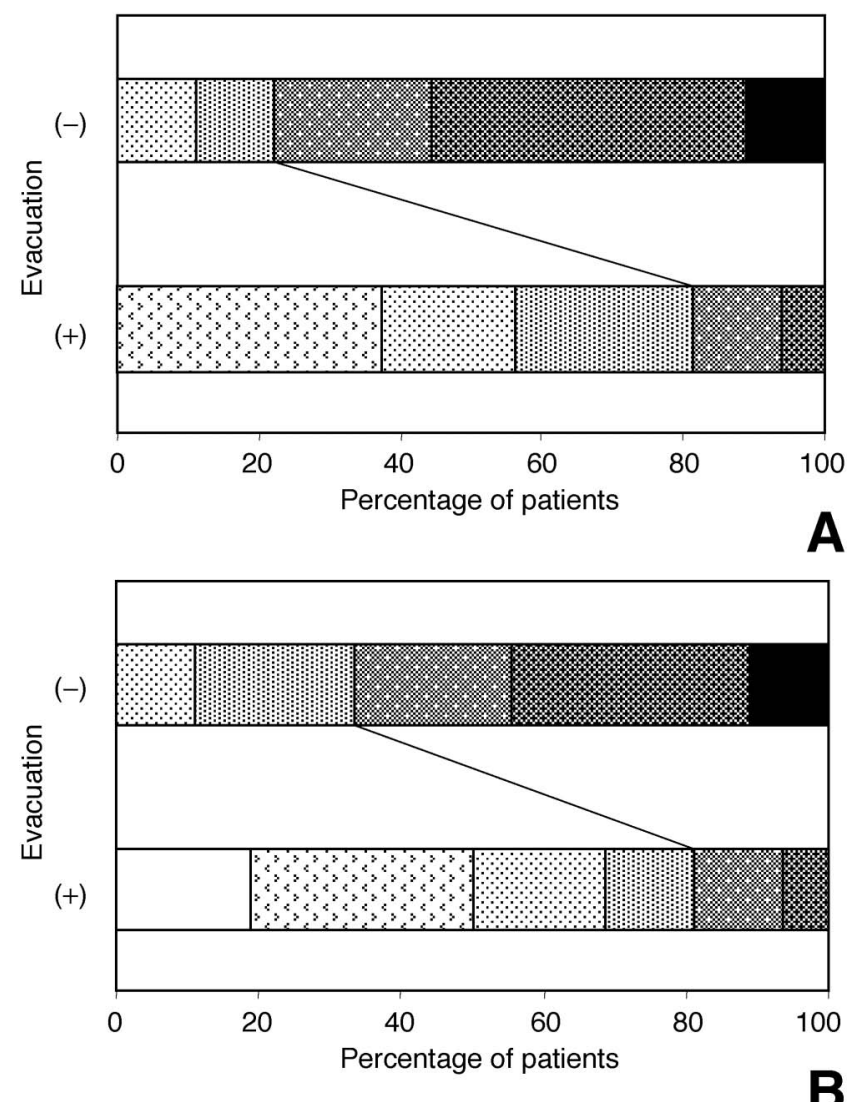

Fig. 6 Functional outcome according to modified Rankin scale (mRS) score after 3 months (A) and 6 months (B). Lines indicate differences between the scores; achievement and non-achievement of hematoma evacuation are defined as $\mathrm{mRS}$ scores 0-3 (good outcome) vs. 4-6 (poor outcome). $\square$ : mRS score 0 , [?: 1, 1

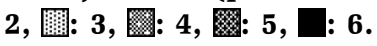

tion (Table 1). Analysis of motor function on the side affected by hematoma and aneurysm rupture according to MMT score at 1 month and functional outcomes determined by mRS score at the 3- and 6month follow up yielded positive findings favoring hematoma evacuation. In patients with any hemiparesis detected during hospitalization, significant improvement of MMT scores ( $p=0.0003$ ) was demonstrated in the group with successful evacuation (Fig. $5)$. Although length of hospital stay did not differ significantly between groups $(p=0.30)$, better functional outcomes as determined by mRS score (0-3) at postoperative 3 months $(p=0.004)$ and 6 months $(p$ $=0.003$ ) were found in patients with successful evacuation (Fig. 6).

\section{Discussion}

Although previous studies have covered a wide range of issues related to the achievement of good overall outcome for patients with sylvian hematoma secondary to rupture of aneurysm, ${ }^{1,15,22,28)}$ little attention has been paid to one potential prognostic factor, mode of clot removal (e.g., microsurgical removal with an irrigation system, surgeon's skills, and extent of evacuation). Patients with sylvian hematoma often develop severe ipsilateral cerebral swelling in the early period (days 1-7 [average, day 4]) after SAH onset. ${ }^{15)}$ This is presumably attributable to vasogenic edema caused by hematoma expansion and surgical manipulation, and may cause the high incidence of vasospasm-induced cerebral infarction. In fact, $82 \%$ of 17 cases of extensive removal of sylvian hematoma developed serious brain edema, which may have been related to surgical maneuvers such as brain retraction or venous injury. As retraction with pial and cortical contusion/disruption is often reported to be an unavoidable consequence in the evacuation of sylvian hematoma, ${ }^{21)}$ effective surgical treatment of these patients remains a great challenge. Sylvian veins compressed by packed hematoma may also contribute to edema formation.

In this study, severe brain edema potentially requiring subsequent decompressive surgery was not encountered even after sylvian hematoma evacuation, and the rate of occurrence of vasospasm-related cerebral infarction was also much lower than in the previous study. ${ }^{15)}$ This might be explained by our consistent strategy of performing extensive removal of hematoma in the early period in conjunction with coagulation of injured subpial vessels to establish adequate reduction of mass effect with minimal injury to surrounding brain tissue. The present findings support the effectiveness of early surgical removal of sylvian hematoma in the management of ICP, microvascular circulation after aneurysm rupture, and associated progression of hematoma.

Our findings suggest that residual hematoma and associated mass effect are important in the etiology of vasospasm and focal neurological deficits following SAH. Massive sylvian hematoma may cause marked mechanical stress resulting in structural changes and biochemical alterations, and thus promote vasospasm at the levels of the vascular endothelium and smooth muscle cells. ${ }^{7,13)}$ Continued release of vasoconstrictive substances from residual hematoma could directly trigger these changes. Successful hematoma evacuation is therefore likely to significantly reduce the incidence of symptomatic vasospasm and associated infarction. The improvements in motor function demonstrated after evacuation suggest that the mass effect of sylvian hematoma is reversible within certain time limits and a 
form of primary brain damage due to aneurysm rupture. Prophylactic decompressive craniectomy, as performed in this study, might be effective in providing immediate and sustained control of ICP in patients with poor WFNS grade after SAH associated with massive sylvian hematoma from MCA aneurysms who were already suffering significant mass effect and brainstem compression at the time of initial surgery. ${ }^{3,22)}$

The outcomes of extensive clot removal as assessed by mRS were found to be encouraging. A good outcome (mRS 0-3) was obtained by 13 of 16 patients $(81 \%)$ at the 6 -month follow up, suggesting that a relatively good quality of life can be expected postrehabilitation in patients who have undergone successful hematoma evacuation. This compares favorably with the results of other studies, ${ }^{3,17,19,21,22,28)}$ even though the majority of our patients did not have a large sylvian hematoma ( $>30 \mathrm{ml}$ volume), which has been reported as an important cutoff point related to outcome of ICH. ${ }^{2,8,14,18)}$ Given that the sylvian hematoma is selfexpanding and associated with bleeding from the subpial vasculature and related cortical injury, early referral with good clinical grade and urgent surgery (time from symptom onset to surgery in the present study was $7.9 \pm 3.6 \mathrm{hrs}$ ) may have contributed to our better functional outcomes. Surgery should comprise extensive clot removal and coagulation of subpial bleeding prior to the development of irreversible brain damage.

There are several limitations to the present study. The retrospective review introduces bias; the sample size is small and so lacks power; and as a single-center study lacks external validity. Thus, our findings might not be directly applicable to other situations or to patients other than the population enrolled (e.g., larger sylvian hematoma). However, when we focus on the direct effect of surgical procedure on treatment outcomes, our data may provide an advantage in evaluating hematoma evacuation itself, since the clinical management for SAH patients throughout the study period strictly adhered to the consistent protocol established at this institution.

In conclusion, our findings indicate that better clinical course and outcomes can be expected in patients who undergo early evacuation of sylvian hematoma with surgical clipping of a ruptured MCA aneurysm. A prospective study is needed to confirm our strategy and to determine further criteria for patient selection.

\section{References}

1) Baskaya MK, Menendez JA, Yuceer N, Polin RS,
Nanda A: Results of surgical treatment of intrasylvian hematomas due to ruptured intracranial aneurysms. Clin Neurol Neurosurg 103: 23-28, 2001

2) Broderick JP, Brott TG, Duldner JE, Tomsick T, Huster G: Volume of intracerebral hemorrhage. A powerful and easy-to-use predictor of 30-day mortality. Stroke 24: 987-993, 1993

3) D'Ambrosio AL, Sughrue ME, Yorgason JG, Mocco JD, Kreiter KT, Mayer SA, McKhann GM 2nd, Connolly ES Jr: Decompressive hemicraniectomy for poor-grade aneurysmal subarachnoid hemorrhage patients with associated intracerebral hemorrhage: clinical outcome and quality of life assessment. Neurosurgery 56: 12-19, 2005

4) Drake C: Report of World Federation of Neurological Surgeons Committee on a Universal Subarachnoid Hemorrhage Grading Scale. J Neurosurg 68: 985-986, 1988

5) Fisher CM, Kistler JP, Davis JM: Relation of cerebral vasospasm to subarachnoid hemorrhage visualized by computerized tomographic scanning. Neurosurgery 6: 1-9, 1980

6) Hadeishi H, Mizuno M, Suzuki A, Yasui N: Hyperdynamic therapy for cerebral vasospasm. Neurol Med Chir (Tokyo) 30: 317-323, 1990

7) Hansen-Schwartz J, Ansar S, Edvinsson L: Cerebral vasoconstriction after subarachnoid hemorrhage-role of changes in vascular receptor phenotype. Front Biosci 13: 2160-2164, 2008

8) Hardemark HG, Wesslen N, Persson L: Influence of clinical factors, CT findings and early management on outcome in supratentorial intracerebral hemorrhage. Cerebrovasc Dis 9: 10-21, 1999

9) Hauerberg J, Eskesen V, Rosenorn J: The prognostic significance of intracerebral haematoma as shown on CT scanning after aneurysmal subarachnoid haemorrhage. Br J Neurosurg 8: 333-339, 1994

10) Heiskanen $O$, Poranen $A$, Kuurne $T$, Valtonen $S$, Kaste M: Acute surgery for intracerebral haematomas caused by rupture of an intracranial arterial aneurysm. A prospective randomized study. Acta Neurochir (Wien) 90: 81-83, 1988

11) Hislop HJ, Montgomery J, Connelly B, Daniels L: Principles of manual muscle testing, in Hislop HJ, Montgomery J (eds): Daniel's and Worthingham's Muscle Testing: Techniques of Manual Examination, ed 6. Oxford, Elsevier Science Health Science div, 1995, pp 2-9

12) Ishikawa $\mathrm{T}$, Kamiyama H, Kobayashi N: [Extensive removal of subarachnoid clot and intracerebral hematoma for poor-grade subarachnoid hemorrhage]. Surgery for Cerebral Stroke 29: 328-334, 2001 (Jpn, with Eng abstract)

13) Keyrouz SG, Diringer MN: Clinical review: Prevention and therapy of vasospasm in subarachnoid hemorrhage. Crit Care 11: 220, 2007

14) Mayer SA, Thomas CE, Diamond BE: Asymmetry of intracranial hemodynamics as an indicator of mass effect in acute intracerebral hemorrhage. A trans- 
cranial Doppler study. Stroke 27: 1788-1792, 1996

15) Niikawa S, Kitajima H, Ohe N, Miwa Y, Ohkuma A: Significance of acute cerebral swelling in patients with sylvian hematoma due to ruptured middle cerebral artery aneurysm, and its management. Neurol Med Chir (Tokyo) 38: 844-850, 1998

16) Nowak G, Schwachenwald D, Schwachenwald R, Kehler U, Muller H, Arnold H: Intracerebral hematomas caused by aneurysm rupture. Experience with 67 cases. Neurosurg Rev 21: 5-9, 1998

17) Prat R, Galeano I: Early surgical treatment of middle cerebral artery aneurysms associated with intracerebral haematoma. Clin Neurol Neurosurg 109: 431-435, 2007

18) Radberg JA, Olsson JE, Radberg CT: Prognostic parameters in spontaneous intracerebral hematomas with special reference to anticoagulant treatment. Stroke 22: 571-576, 1991

19) Schirmer CM, Hoit DA, Malek AM: Decompressive hemicraniectomy for the treatment of intractable intracranial hypertension after aneurysmal subarachnoid hemorrhage. Stroke 38: 987-992, 2007

20) Seiler RW, Newell DW: Subarachnoid hemorrhage and vasospasm, in Newell DW, Aaslid R (eds): Transcranial Doppler Sonography. New York, Raven Press, 1992, pp 101-107

21) Shimoda M, Oda S, Mamata Y, Tsugane R, Sato O: Surgical indications in patients with an intracerebral hemorrhage due to ruptured middle cerebral artery aneurysm. J Neurosurg 87: 170-175, 1997

22) Smith ER, Carter BS, Ogilvy CS: Proposed use of prophylactic decompressive craniectomy in poorgrade aneurysmal subarachnoid hemorrhage patients presenting with associated large sylvian hematomas. Neurosurgery 51: 117-124, 2002

23) Su CC, Saito K, Nakagawa A, Endo T, Suzuki Y, Shirane $\mathrm{R}$ : Clinical outcome following ultra-early operation for patients with intracerebral hematoma from aneurysm rupture-focussing on the massive intrasylvian type of subarachnoid hemorrhage. Acta Neurochir Suppl 82: 65-69, 2002

24) Tanikawa R: [Less invasive cisternal approach and removal of subarachnoid hematoma for the treatment of ruptured cerebral aneurysms]. No Shinkei Geka 35: 17-24, 2007 (Jpn, with Eng abstract)

25) Tokuda $Y$, Inagawa $T$, Katoh $Y$, Kumano K, Ohbayashi N, Yoshioka $\mathrm{H}$ : Intracerebral hematoma in patients with ruptured cerebral aneurysms. Surg Neurol 43: 272-277, 1995

26) van Swieten JC, Koudstaal PJ, Visser MC, Schouten $\mathrm{HJ}$, van Gijn J: Interobserver agreement for the assessment of handicap in stroke patients. Stroke 19: 604-607, 1988

27) Yamaguchi M, Bun T, Kuwahara T, Kitamura S: Very late-onset symptomatic cerebral vasospasm caused by a large residual aneurysmal subarachnoid hematoma-case report. Neurol Med Chir (Tokyo) 39: 677-680, 1999

28) Yoshimoto Y, Wakai S, Satoh A, Hirose Y: In- traparenchymal and intrasylvian haematomas secondary to ruptured middle cerebral artery aneurysms: prognostic factors and therapeutic considerations. Br J Neurosurg 13: 18-24, 1999

Address reprint requests to: Tatsushi Mutoh, M.D., Department of Surgical Neurology, Research Institute for Brain and Blood Vessels-Akita, 6-10 SenshuKubota-machi, Akita 010-0874, Japan.

e-mail:tmutoh@tiara.ocn.ne.jp

\section{Commentary}

The authors have to be congratulated on presenting a series of patients with SAH and intra-axial hematoma. More than 20 years ago, we started with operating ruptured aneurysms in the acute stage. Ever since, it has been clear that evacuation of the blood clot from subarachnoid space (SAS) and/or from the intra-axial compartment around the aneurysm(s) is an equally important part of surgery as is clipping of the aneurysm itself. It is clear that one cannot have an influence on the initial brain damage due to SAH and resulting hematoma, but with complete removal of the hematoma and correct clipping of the aneurysm by preservation of the blood flow, the circulation of the CSF is reestablished, and the blood vessels handled accordingly will also develop much less vasospasm than without appropriate extirpation of the blood clot from the SAS. In addition, personally, I have been convinced that with opening of the third ventricle by cutting the lamina terminalis, the circulation of the CSF can be further improved and the rest of the blood in the SAS is more successfully washed-out. Whatever is the cause of vasospasm, there is no doubt that degrading products of extravasated blood do change the $\mathrm{pH}$ of the CSF, which additionally does affect the adventitia innervation of the intracranial blood vessels. Of course, the factor of time is also important; the sooner the better for evacuation of the blood clot. There can be no doubt additional supportive treatment for such patients is necessary and is very beneficial. Now the question is how far one can go, and which is the initial status that still promises a good outcome. My practical answer will be-instead of waiting for amelioration of the initial status of a patient with a ruptured aneurysm and large blood clot, it is much better to act as soon as possible.

The authors are talking about brain edema; however, I have to admit that - also thanks to good neuroanesthesia-personally I did not encounter such a problem when operating ruptured aneurysms in the acute stage. Personally, I have operated more 
than 500 patients with ruptured aneurysms in the acute stage and in most of the cases after removal of the hematoma, clipping the aneurysm, and opening of the lamina terminalis, and washing out the hematoma, the brain was relaxed. Never ever did I encounter a situation which I could not deal with after removal of a hematoma and the clot from the basal cisterns. From our experience, I do favor early surgery for all aneurysm ruptures; in particular in those cases where there is a significant clot in the skull base or intra-axially. In addition, it has to be mentioned that the patients with intra-axial blood clot and with a massive $\mathrm{SAH}$ at the skull base are certainly not candidates for endovascular treatment.

Vinko V. DOLEnC, M.D. Department of Neurosurgery University Medical Center Ljubljana, Slovenia
The authors propose a strategy to improve the treatment outcome of subarachnoid hemorrhage patients with sylvian hematoma associated with ruptured MCA aneurysms, comprising early surgical evacuation prior to hematoma expansion; extensive removal of any dense subarachnoid and subpial clots; and coagulation of bleeding pial vessels injured by the impact of aneurysm rupture, and found that the frequencies of delayed ischemic neurological deficit and vasospasm-related cerebral infarction were significantly less in the extensive hematoma evacuation achievement group. The clinical data is very detailed and the conclusion is reliable.

Ya-zhuo Zhang, M.D. Deputy Director Beijing Neurosurgical Institute Beijing, P.R.C. 